\title{
Mitarbeitende gewinnen, Mitarbeitende fördern
}

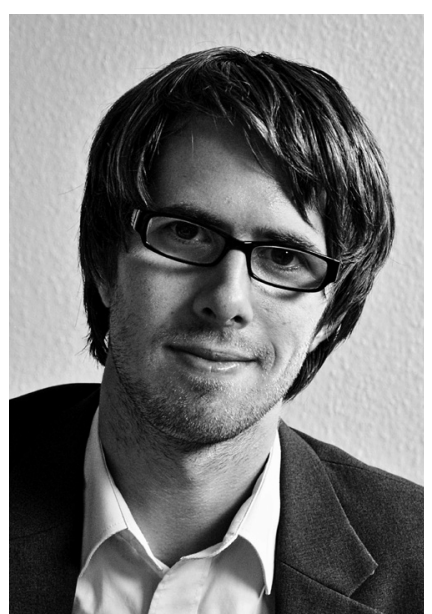

VON MATTHIAS HARTKAMP Matthias Hartkamp leitete in den vergangenen zwei Jahren das Rückenwindprojekt "beneficial place of work - gemeinsam den Wandel gestalten « der Benediktushof gGmbH. Das hier beschriebene Personalentwicklungskonzept ist ein Ergebnis des unternehmerischen Projektes. Das Projekt wurde im Rahmen des Programms »rückenwind - Für die Beschäftigten in der Sozialwirtschaft» durch das Bundesministerium für Arbeit und Soziales und den Europäischen Sozialfonds gefördert. www.benediktushof.de

\author{
In einem Projekt innerhalb des Bundesprogramms \\ "Rückenwind" wurde ein Gesamtkonzept zur \\ Personalentwicklung in Zeiten des Fachkräftemangels \\ entwickelt, das praktische Anregungen für soziale \\ Organisationen und Unternehmen bieten kann.
}

$\mathrm{Zu}$ Beginn des Konzeptes steht die Personalgewinnung im Mittelpunkt. Durch ein zentrales Bewerbermanagement wird eine zeitnahe Rückmeldung auf die eingehenden Bewerbungen gewährleistet. Eine Bewerberdatenbank ermöglicht es außerdem, zu jedem Zeitpunkt eine Aussage über den aktuellen Bearbeitungsstand sowie den aktuellen Ansprechpartner zu tätigen.

Die Zusammenarbeit mit berufsbildenden Schulen und Hochschulen gewährleistet eine enge Anbindung an die Berufsausbildung und fördert die Verknüpfung zwischen neuen Entwicklungen in den Bereichen Theorie, Forschung und Praxis. Wenn eine vakante Stelle durch einen neuen Mitarbeiter erfolgreich besetzt wurde, stellt die Einführung des neuen Mitarbeiters die nächste Stufe im Personalentwicklungskonzept dar. Hierfür setzt der Benediktushof Maria Veen die folgenden Instrumente ein: Am ersten Arbeitstag erhalten alle neuen Mitarbeiter eine Willkommensmappe von ihrem Vorgesetzten überreicht. In dieser befinden sich ein personalisierter Willkommensgruß der Geschäftsführung sowie die wichtigsten Informationen über das Unternehmen und eine kleine »Aufmerksamkeit«. Ursprung dieser Mappe war eine Begrüßungsmappe in einem Hotel, welche zur Begrüßung auf den Gast im Zimmer wartet und als erste Orientierungshilfe dient.

Abteilungsspezifische Einarbeitungspläne, die auf die Besonderheiten am jeweiligen Arbeitsplatz eingehen und gleichzeitig den Zusammenhang zum gesamten Betrieb aufzeigen, gewährleisten eine detaillierte und fundierte Einarbei- tung. Insbesondere die Zeit in den ersten Wochen ist entscheidend für den langfristigen Verbleib und Erfolg des neuen Kollegen im Unternehmen. Gerade in dieser Phase kann durch ein wertschätzendes, herzliches Willkommen eine frühzeitige emotionale Bindung und Beziehung an das Unternehmen forciert werden.

Das nächste Kapitel des Konzeptes legt den Fokus auf die tägliche Mitarbeiterführung. Im Rahmen von drei ganztägigen Workshops entwickelten die Führungskräfte des Benediktushofs gemeinsam mit der Projektleitung die unternehmerischen Führungsgrundsätze. Aufgeteilt in die vier Dimensionen: "So führen wir", »Transparenz«, »Vorbild « und »Verantwortung « stellen 25 Grundsätze die Spielregeln für den gemeinsamen Umgang dar. Das Einhalten dieser Spielregeln kann in der täglichen Arbeit von Mitarbeitern und Führungskräften gleichermaßen eingefordert werden.

Einen wesentlichen Bestandteil des Rückenwind-Projektes bildete die Qualifizierung aller Führungskräfte in vier aufeinander aufbauenden Modulen. Die Seminare behandelten Themen wie wertschätzende Kommunikation, gesunde Führung, Generationen-Management sowie Diversity-Management, das heißt: das Führen individuell verschiedener Mitarbeiter. Um auch den Blick über den Tellerrand hinaus zu ermöglichen, erfolgte die Qualifizierung in Kooperation mit den Caritasverbänden Coesfeld und Gelsenkirchen. Beide Institutionen stellten Mitarbeiter frei, die an den insgesamt zehn Qualifizierungstagen teilnahmen. Um einen besseren Lernerfolg und ein 
intensiveres Arbeiten zu gewährleisten, fand die Qualifizierung in Kleingruppen mit zehn bis zwölf Personen statt.

Ausgehend von den Ergebnissen einer Mitarbeiterbefragung zu Projektbeginn sind die Kamingespräche im Benediktushof entstanden. Diese quartalsweisen Gespräche - ursprünglich im »Kaminzimmer« des Unternehmens angesiedelt bieten allen Mitarbeitern die Möglichkeit, mit den Mitgliedern des Leitungsteams ins Gespräch zu kommen. Anders als in vielen Konferenzen gibt es keine feste Tagesordnung und kein festes Thema. Vielmehr stehen der Austausch und die bereichsübergreifende Weitergabe von Informationen im Vordergrund. So wird ein Forum des unkomplizierten Miteinanders geschaffen. Nach rund eineinhalb Jahren sind die Kamingespräche langsam in der Unternehmenskultur verstetigt und erfreuen sich einer wachsenden Beliebtheit.

Ausgehend von den Jahreszielen des Unternehmens und in Verbindung mit den Ergebnissen werden individuelle Qualifizierungsbedarfe der Mitarbeiter ermittelt. Die Personalentwicklung sammelt die unterschiedlichen Bildungsbedarfe und prüft, ob es vorteilhaft ist, das Seminar intern anzubieten oder externe Bildungsangebote zu nutzen. Für diese Entscheidungsfindung stellen die Verfügbarkeit von Bildungsangeboten sowie die Gruppengröße zwei wichtige Indikatoren dar. Typische Themenfelder für Inhouse-Seminare sind beispielsweise Medikamentenkunde, Behinderungsbilder, Medizinprodukte; aber auch Angebote zum Zeitmanagement oder zum gesunden Arbeiten.

Mit Hilfe von Personalentwicklungsplänen zeigt der Benediktushof seinen Mitarbeitern frühzeitig Entwicklungsperspektiven im Unternehmen auf. Im Vorfeld einer neu zu besetzenden Stelle erarbeiten Personalentwicklung und die zuständige Abteilungsleitung gemeinsam den Entwicklungsplan und legen individuelle Meilensteine fest. Diese Pläne zeigen sowohl horizontale als auch vertikale Karriereoptionen auf und zeigen einen Planungshorizont von 12 bis 24 Monaten auf.

Nicht zuletzt durch den wachsenden Fachkräftemangel rückt der Fokus verstärkt darauf, die Arbeitsfähigkeit der Mitarbeiter fördern und erhalten. Das in den letzten zwei Jahren eingeführte betriebliche Gesundheitsmanagement hat sich die Erhaltung der Mitarbeitergesundheit zum Ziel gesetzt. So sind darin

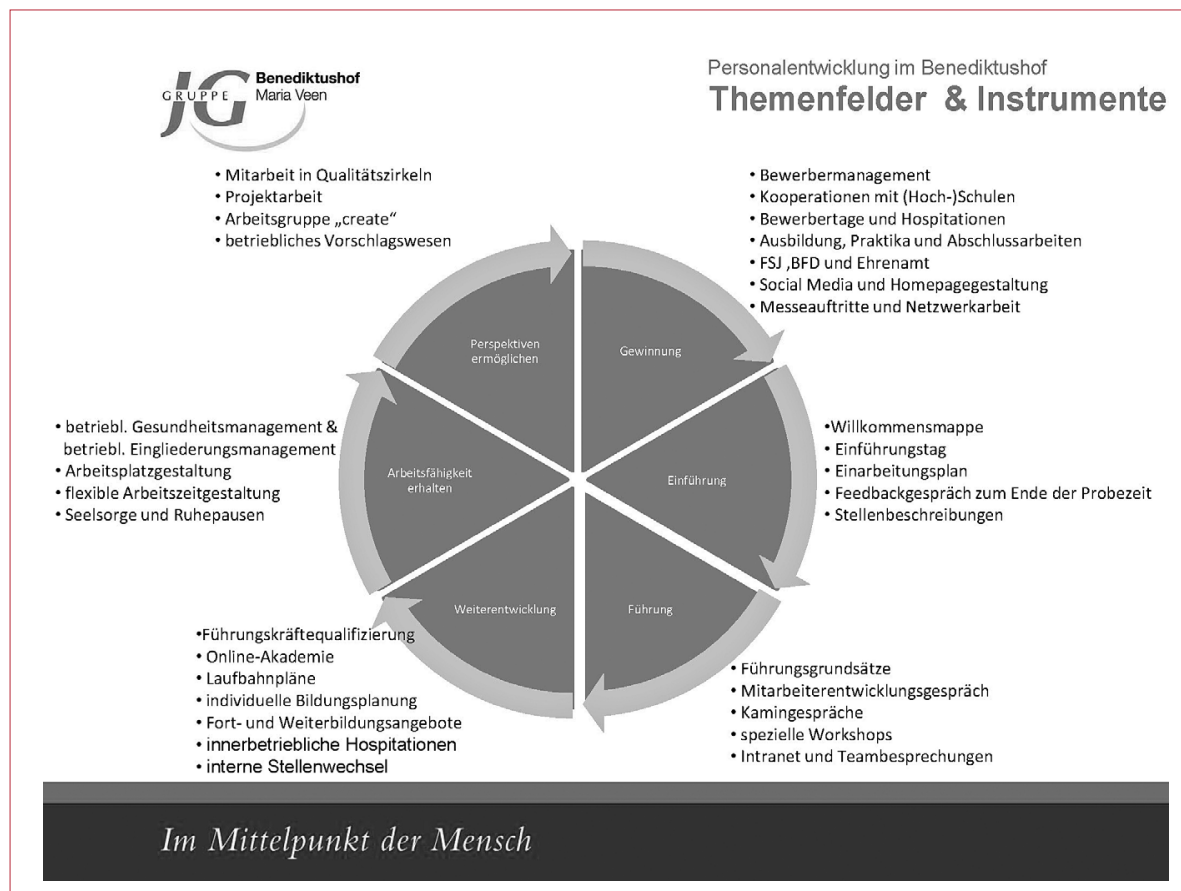

Für ein stimmiges, an das jeweilige Unternehmen angepasste Konzept der Personalentwicklung stehen unterschiedliche Instrumente zur Verfügung.

sowohl das betrieblichen Eingliederungsmanagement als auch Maßnahmen der betrieblichen Gesundheitsförderung sowie umfangreiche Analysen und Auswertungen zusammengefasst. Das differenzierte Portfolio umfasst Maßnahmen aus den Bereichen Ruhe und Entspannung, Bewegung und Fitness, Ergonomie sowie Fortbildungen zu gesundheitsbezogenen Themen. Zu beachten ist, dass sich das betriebliche Gesundheitsmanagement nicht vorrangig an den einzelnen Mitarbeiter, sondern an das System Betrieb richtet und der Gesundheitsgedanken dadurch in den betrieblichen Prozess eingebunden ist. Die Verankerung der Gesundheitsorientierung in den Führungsgrundsätzen des Unternehmens spiegelt die innerbetriebliche Bedeutung des Gesundheitsmanagements wider.

Die letzte Dimension des unternehmerischen Konzeptes stellt der Aspekt "neue Perspektiven" dar. Vorrangiges Ziel dieser Maßnahmen ist, die Motivation der langjährigen Mitarbeiter zu erhalten und die Entwicklungsbereitschaft zu fördern. Durch innerbetriebliche Hospitationen erwerben die Mitarbeiter neue Einblicke in andere Tätigkeitsfelder des Unternehmens und können so potenzielle neue Arbeitsbereiche für sich entdecken. Gleichzeitig wird der Austausch untereinander gefördert und somit auch das Verständnis für andere Tätigkeitsfelder erhöht.
Im Rahmen von verschiedenen Qualitätszirkeln erhalten die Mitarbeiter die Möglichkeit, sich aktiv in die Unternehmensentwicklung einzubringen und neue Perspektiven für sich und das Arbeitsfeld zu entdecken. Die berufsgruppenübergreifend besetzten Qualitätszirkel arbeiten themenspezifisch direkt der Geschäftsführung zu und geben so Anregungen aus der Praxis. Qualitätszirkel bestehen beispielsweise $\mathrm{zu}$ den Themen Sozialraumorientierung, technische Assistenz, Ethik oder Gesundheitsmanagement, wobei es für jeden Zirkel einen vorab bestimmten Verantwortlichen gibt.

\section{Resümee}

Bei der Vielzahl der initiierten Maßnahmen ist eine gute Abstimmung und Koordination der einzelnen Dimensionen unverzichtbar. Vor diesem Hintergrund wurde die Stelle der Projektleitung zum Projektende in eine Stabsstelle Personalentwicklung umgewandelt. Zentrale Aufgabe dieser neuen Stelle ist es, die einzelnen Maßnahmen in ihren unterschiedlichen Ausprägungen zu verstetigen. Eine besondere Herausforderung hierbei stellt die Abstimmung mit den unterschiedlichen Mitarbeitern und Bereichen dar, da je nach Instrument andere Kollegen und Zielgruppen eingebunden sind. 